\title{
Assessment of correlation between knee notch width index and the three-dimensional notch volume
}

\author{
Carola F. van Eck - Cesar A. Q. Martins • \\ Stephan G. F. Lorenz $\cdot$ Freddie H. Fu • \\ P. Smolinski
}

Received: 11 February 2010/ Accepted: 16 March 2010/Published online: 8 April 2010

(c) The Author(s) 2010. This article is published with open access at Springerlink.com

\begin{abstract}
This study was done to determine whether there is a correlation between the notch volume and the notch width index (NWI) as measured on the three most frequently used radiographic views: the Holmblad $45^{\circ}$, Holmblad $70^{\circ}$, and Rosenberg view. The notch volume of 20 cadaveric knees was measured using Computed Tomography (CT). The Holmblad $45^{\circ}$, Holmblad $70^{\circ}$, and Rosenberg notch view radiographs were digitally re-created from the CT scans for each specimen, and the NWI was measured by two observers. The Pearson correlation coefficient between the NWI and notch volume was calculated, as well as between the three views. An independent $t$ test was performed to determine the difference in NWI and notch volume between male and female specimens. The reliability for each view was also determined. There was no correlation between the NWI as measured on the Holmblad $45^{\circ}$, Holmblad $70^{\circ}$, or Rosenberg view and the notch volume. All three radiographic views proved reliable, but showed only a moderate correlation with each other. Men had larger notch volumes than women, but there was no difference in NWI. A knee with a small intercondylar notch is often considered an increased risk for ACL rupture. The NWI is a frequently used two-dimensional method to
\end{abstract}

C. F. van Eck - C. A. Q. Martins · S. G. F. Lorenz ·

F. H. Fu ( $)$ - P. Smolinski

Department of Orthopaedic Surgery, University of Pittsburgh

Medical Center, Kaufman Building Suite 1011,

3471 Fifth Avenue, Pittsburgh, PA 15213, USA

e-mail: ffu@upmc.edu

C. F. van Eck

Orthopaedic Research Centre Amsterdam,

Academic Medical Centre, Department G4, Meibergdreef 9,

1100 DD Amsterdam, The Netherlands determine notch size. However, in the present study, this index was not positively correlated with the overall volume of the notch. Based on the results of the current study, the authors would advice to use caution when using notch view radiographs in a clinical setting to predict risk of ACL rupture.

Keywords Anterior cruciate ligament .

Notch width index · Intercondylar notch · Radiograph . Computed Tomography

\section{Introduction}

The close relationship between the function of the anterior cruciate ligament (ACL) and the femoral intercondylar notch has long been recognized [10]. The most widely used two-dimensional measurement of the notch size is the notch width index (NWI). This method was described by Souryal and Freeman in 1988 and is defined as the width of the intercondylar notch at the level of the popliteal groove divided by the bicondylar width at the same level [13]. This is measured on a notch view radiograph with the knee in $45^{\circ}$ of flexion. After the NWI was established, many different notch view radiographic methods have been proposed to visualize the anterior and posterior outlet of the notch and the popliteal groove: the Holmblad $45^{\circ}$ view [7], the Holmblad $70^{\circ}$ view $[8,12]$, and the Rosenberg view $[6,11]$. The accuracy of these methods was recently studied by Anderson et al., who compared the radiographic measurements to caliper measurements in cadaveric specimens and concluded that only the Holmblad $70^{\circ}$ view was accurate [1]. However, to our knowledge, there has not been any study to determine which (if any) radiographic view best predicts overall notch size (i.e., volume). 
The primary aim of the present study was to determine the correlation between the three-dimensional notch volume and the NWI as measured on the three most frequently used radiographic views: the Holmblad $45^{\circ}$, Holmblad $70^{\circ}$, and Rosenberg view.

We also had three secondary aims. The first was to determine the intra- and inter-observer reliability of the three views. The second to determine the correlation between the three NWIs as measured on the Holmblad $45^{\circ}$, Holmblad $70^{\circ}$, and Rosenberg view. Lastly, we aimed to determine whether there was a difference in NWI and notch volume between male and female specimens.

\section{Methods and materials}

This was a controlled laboratory study. Twenty cadaveric knees underwent computed tomography (CT) imaging of the knee. Specimens were excluded from the study when osteoarthritic changes were seen on the CT scan. The boundaries of the intercondylar notch were defined based on anatomic landmarks. The superior starting point of the notch was defined as the first axial slice to show both condyles. This was confirmed on the sagittal view. The inferior endpoint of the notch was defined as the last axial slice with continuity between the medial and the lateral femoral condyle. The posterior border of the notch in each axial image was said to be the line drawn between the two points on the inside of the femoral condyles with a sudden change of slope. This method was based on the technique by Charlton et al. [3] for MRI . This method was first validated through intra- and inter-observer testing and found to be highly reliable. Manual digital tracings of the notch area were performed on the axial CT images, using Osirix (Osirix, Geneva, Switzerland), 3D imaging software. The measured areas were then used to calculate the volume by summing the number of images within the defined femoral notch and multiplying by the slice thickness (Fig. 1).

Digitally reconstructed radiographs were created from the CT scans using Amira software (Visage Imaging $\mathrm{GmbH}$, Berlin, Germany). This software allows for digitally reconstructed radiographs to be made from the data of the CT scan, simulating different X-ray source positions and angles (Fig. 2). First, all femurs were aligned in a way that the posterior condyles were superimposed in the sagittal orientation. Subsequently, the Holmblad $45^{\circ}$, Holmblad $70^{\circ}$, and Rosenberg view were simulated according to the description of the methods by Anderson et al. [1] to create a notch view radiograph for all the cadaveric knees for the three different methods (Fig. 3). The NWI was measured according to the method by Souryal and Freeman [13], using ImageJ (ImageJ v1.41o, National Institutes of Health, USA). The width of the intercondylar notch at the level of the popliteal groove was measured and divided by the bicondylar width at the same level (Fig. 4). Two observers measured the NWI twice on two separate occasions one week apart. One of the observers was a research fellow in orthopedic surgery working on a $\mathrm{PhD}$ thesis, and the second observer a sports medicine trained orthopedic surgeon with 15 years experience.

\section{Statistical analysis}

The data were analyzed with PASW Statistics (version 17.0, SPSS Inc., Chicago, IL). The intra-class correlation coefficient (ICC) was calculated to determine both intra- and interobserver reliability for the NWI as measured on the three different views. The measurements of one observer were used for further statistical analysis. The Pearson correlation coefficient was calculated to determine the correlation between the NWI measured on the Holmblad $45^{\circ}$, Holmblad $70^{\circ}$, and the Rosenberg view. The Pearson correlation coefficient was also used to determine the correlation between the three NWIs and the notch volume. An independent $t$ test was performed to determine the difference in NWI on all three views and notch volume between male and female specimens. An alpha level of 0.05 was chosen for statistical significance. Sample size was not calculated before starting the study, due to the absence of a reference range of the expected correlation between the NWI and notch volume. Therefore, a post hoc power analysis was performed.

\section{Results}

The demographic data of the 20 cadaveric specimens are displayed in Table 1. The ICC for the NWI measurements on the three radiographic views for intra-rater reliability ranged from 0.81 to 0.98 and for inter-rater reliability from 0.89 to 0.94 . All the ICC's and their $95 \%$ confidence intervals are displayed in Table 2. The measured NWI ranged from 0.24 to 0.38 . The Rosenberg view displayed the largest range, while the Holmblad $70^{\circ}$ had the smallest range. The average notch volume was $7.3 \mathrm{~cm}^{3}$ and ranged from 3.5 to $18.2 \mathrm{~cm}^{3}$ (Table 3 ). The correlation between the NWI measured on the Holmblad $45^{\circ}$ view and the Holmblad $70^{\circ}$ view was $0.78(P<0.001)$, between the Holmblad $45^{\circ}$ and the Rosenberg view was 0.71 $(P<0.001)$, and between the Holmblad $70^{\circ}$ and Rosenberg view was $0.66(P=0.002)$. There was no significant correlation between the notch volume and the NWI as measured on either the Holmblad $45^{\circ}$, Holmblad $70^{\circ}$, or Rosenberg view. The NWI as measured on the Holmblad 


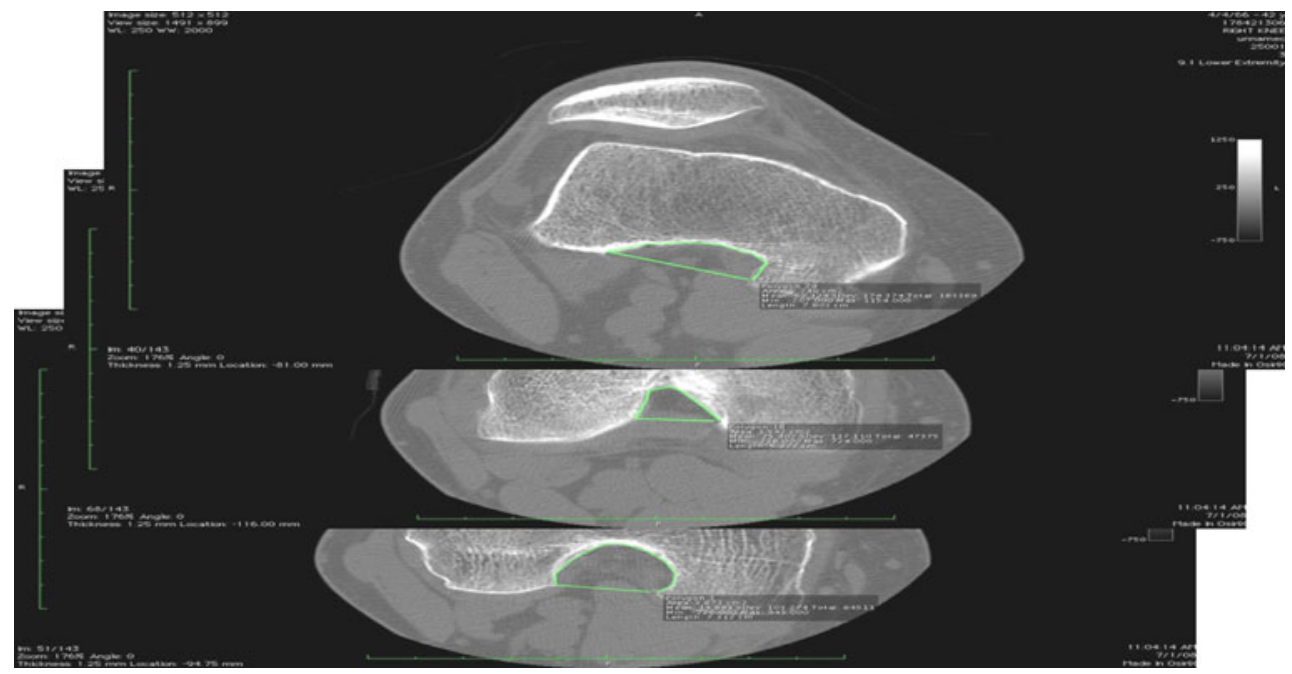

Fig. 1 The notch area was measured on all axial CT slices that contained the notch. The green outline illustrates the notch area, and the box provides information about the size. Subsequently, all the areas were summated and multiplied by the slice thickness to determine the notch volume. This is a simplified image, since an average notch would contain between 30 and 100 slices, depending on the extent of the notch

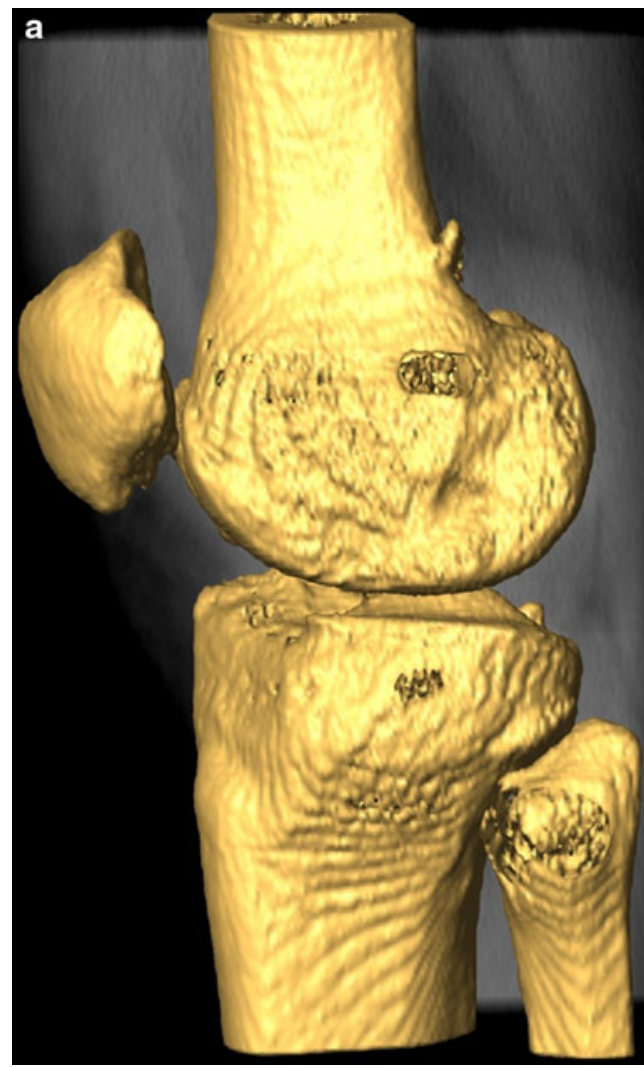

Fig. 2 This image illustrates how the Amira software reconstructs a digital radiograph, using a three-dimensional reconstruction of the CT scan. a The three-dimensional reconstruction of the CT scan of the knee is made, and a digital radiograph is made through this reconstruction and projected on the background. b The femur is rotated to simulate the position to the X-ray source according to the

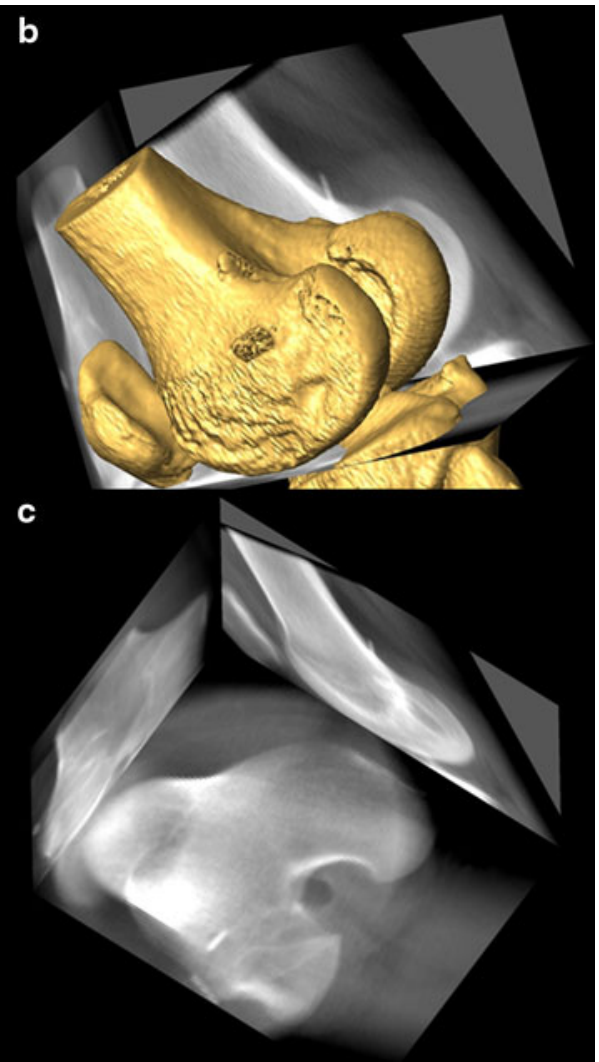

three different notch view techniques. The tibia is partially removed to eliminate over-projection and improve image quality of the simulated radiograph $\mathrm{C}$. The reconstructed CT scan is removed and the notch view radiograph is visible on the bottom. This notch view radiograph can be saved as a separate image and subsequently measured with ImageJ software 

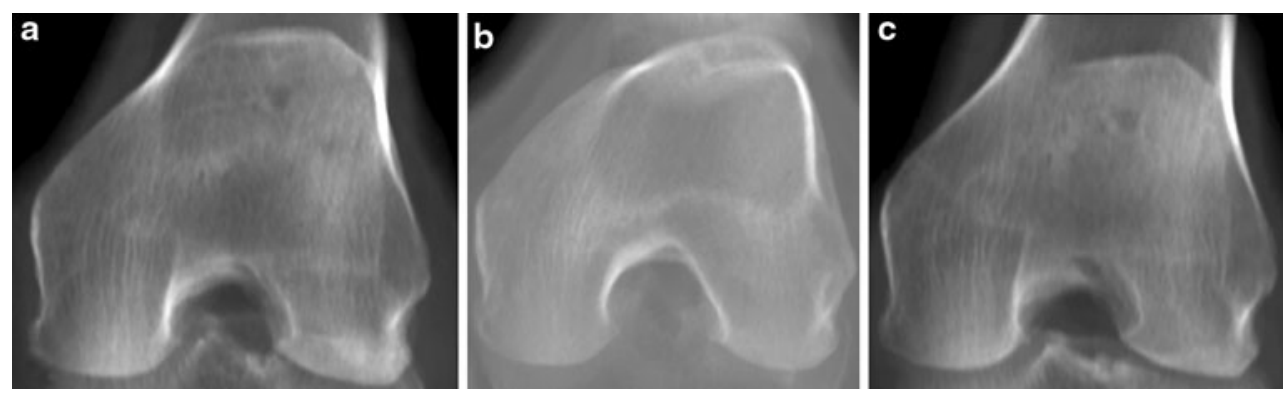

Fig. 3 Example of the digitally reconstructed radiographs from the CT scan with Amira software for one of the specimens. a Holmblad $45^{\circ}$ view. b Holmblad $70^{\circ}$ view. c Rosenberg view

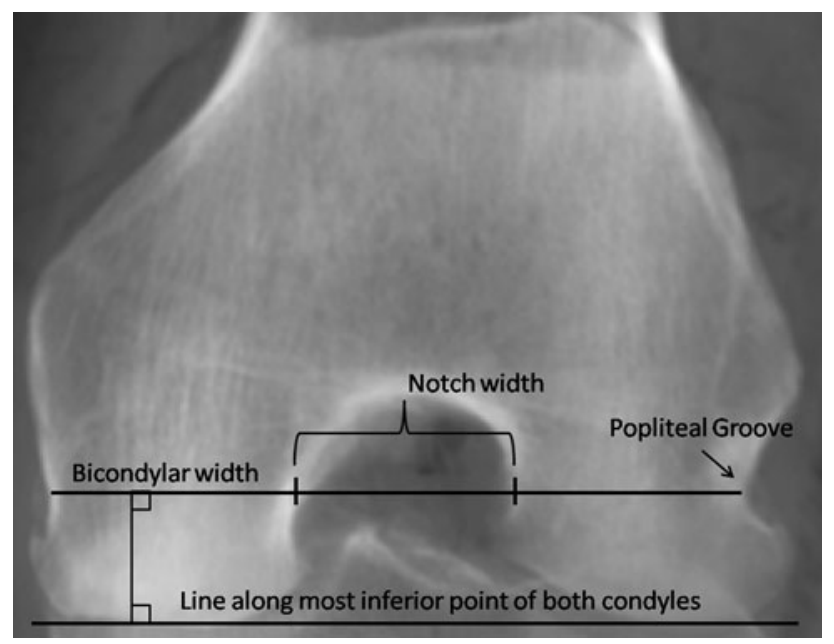

Fig. 4 Method to measure the notch width index. A line is drawn between the most inferior points of both condyles. The bicondylar width is measured along this line, at the level of the popliteal groove. Lastly, the notch width is measured at this same level

$45^{\circ}$, Holmblad $70^{\circ}$, and Rosenberg view was not different between male and female specimens. There was a significant difference in notch volume between the men and women $(P=0.003)$ (Table 4$)$.

\section{Discussion}

The main finding of the present study was the absence of a statistically significant correlation between the NWI and

Table 1 Demographics of the 20 cadaveric specimens

\begin{tabular}{llrc}
\hline & Mean or ratio & SD & Range \\
\hline Gender:male:female & $10: 10$ & & \\
Age (years) & 58 & 7 & $37-67$ \\
Height (cm) & 173 & 10 & $155-191$ \\
Weight $(\mathrm{kg})$ & 70 & 19 & $44-108$ \\
Side:right:left & $10: 10$ & & \\
\hline
\end{tabular}

$S D$ standard deviation
3D notch volume. The primary aim of this study was to determine whether there is a correlation between the notch volume and the NWI as measured on the three most frequently used radiographic views: the Holmblad $45^{\circ}$, Holmblad $70^{\circ}$, and Rosenberg view, and which view has the best correlation with notch volume. This was attempted in order to see if NWI could predict overall notch size. A reason for the absence of a significant correlation could be that the NWI is an index that corrects a patient's notch width for his or her bicondylar width, while the overall notch volume is an absolute measurement, not corrected for the patients' bone size.

The average NWI in this study was 0.31 . This is slightly higher than the average NWI in previously published papers. Souryal et al. [12, 13] described a mean NWI of 0.23, Anderson et al. [1] 0.26, and Chandrashekar et al. [2] was the closest to the current study with 0.28 . This illustrates that there is a wide range of both notch width and bicondylar width. Differences could be based on the difference in age between the studied populations.

One of our secondary aims was to determine whether there was a difference between male and female specimens with respect to NWI and volume. We found no difference in NWI on either the Holmblad $45^{\circ}$, Holmblad $70^{\circ}$, or Rosenberg view between. However, there was a significantly larger notch volume in the male subjects. This is in line with other literature that finds a larger notch size in men than in women $[3-5,9,14]$.

Another secondary aim was to determine the intra- and inter-observer reliability of the Holmblad $45^{\circ}$, Holmblad $70^{\circ}$, and Rosenberg view. A good intra- and inter-observer reliability was found for all three views. The Holmblad $45^{\circ}$ had the best combination of a good intra- and interobserver reliability. A possible explanation for this could be that this view has good visualization of both the notch outlet, as well as the popliteal groove, which results in consistency of the measurement location.

The last secondary aim was to determine the correlation between the three NWI as measured on the Holmblad $45^{\circ}$, Holmblad $70^{\circ}$, and Rosenberg view. There was a moderate 
Table 2 Intra-class correlation coefficients for intra- and inter-rater reliability

\begin{tabular}{llll}
\hline & ICC rater 1 & ICC rater 2 & ICC rater 1 versus 2 \\
\hline NWI Holmblad 45 & $0.93[0.80-0.98]$ & $0.91[0.64-0.97]$ & $0.94[0.66-0.98]$ \\
NWI Holmblad 70 & $0.81[0.52-0.93]$ & $0.93[0.81-0.97]$ & $0.90[0.74-0.96]$ \\
NWI Rosenberg & $0.98[0.94-0.99]$ & $0.88[0.70-0.95]$ & $0.89[0.58-0.96]$ \\
\hline
\end{tabular}

ICC intra-class correlation coefficient, NWI notch width index. [95\% Confidence Interval]

Table 3 Measured notch width indexes and notch volumes

\begin{tabular}{llll}
\hline & Mean & SD & Range \\
\hline NWI Holmblad 45 & 0.31 & 0.03 & $0.26-0.38$ \\
NWI Holmblad 70 & 0.32 & 0.03 & $0.27-0.38$ \\
NWI Rosenberg & 0.30 & 0.04 & $0.24-0.38$ \\
Notch volume $\left(\mathrm{cm}^{3}\right)$ & 7.3 & 3.5 & $3.5-18.2$ \\
\hline
\end{tabular}

$S D$ standard deviation, $N W I$ notch width index

Table 4 Difference in NWI and notch volume between male and female specimens

\begin{tabular}{llll}
\hline & Mean males & Mean females & $P$ value \\
\hline NWI Holmblad 45 & 0.31 & 0.31 & NS \\
NWI Holmblad 70 & 0.32 & 0.32 & NS \\
NWI Rosenberg & 0.30 & 0.31 & NS \\
Notch volume $\left(\mathrm{cm}^{3}\right)$ & 9.5 & 5.1 & 0.003 \\
\hline
\end{tabular}

NWI notch width index

to good correlation between the three methods, ranging from 0.66 to 0.78 . This translates to about $50 \%$ overlap between the different methods ( $r^{2}$ between 0.44 and 0.60 ). This can be explained by the different measurement locations, caused by the different views. The three radiographic methods each result in a different view of the notch and a different part of the notch outlet that is in line with the

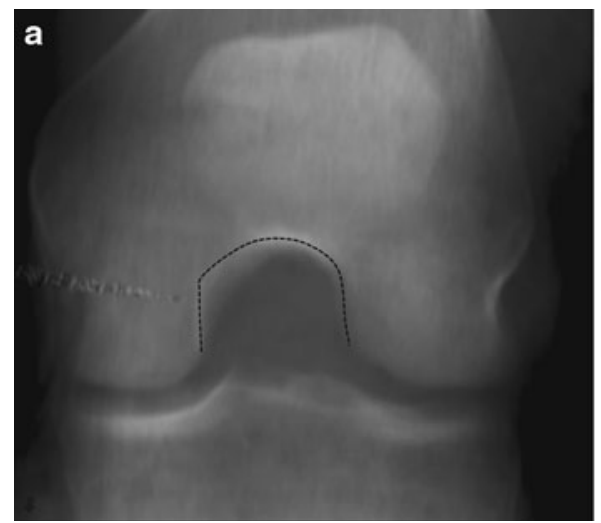

Fig. 5 The notch has a variety of different shapes. Two Holmblad $45^{\circ}$ notch view radiographs are shown of two different specimens. a Round-shaped notch; variation in the measurement location in this specimen would not cause a large difference in notch width index, popliteal groove. The anatomic variability of the notch, in particular the notch shape, determines the magnitude of the effect of using the different views. If the notch is more A-shaped, measuring at a different location in the notch has a larger effect than if the notch has a more rounded shape (Fig. 5).

The limitations of this study are that CT scans were used to reconstruct digital radiographs in different configurations, rather than using living subjects and taking radiographs of their knees. The method used in this study is more consistent because it uses a standardized protocol that is not influenced by compliance and alignment of the patient and accidentally rotated radiographs. This degree of accuracy may, however, be hard to reproduce in a hospital setting, using the different notch view radiographic configurations, and therefore the reliability of the measurements may be overestimated in the present study. Furthermore, flexion was simulated by rotation of the femur relative to the digital X-ray source after that the tibia was partially removed to eliminate overlap and improve image quality. In the current study, we were not able to compare the measured notch volume and NWI to actual measurements taken from the cadaveric specimens. This was due to that the cadaveric specimens had to be used for another study as well and therefore were not available for dissection. However, correlation between radiographic

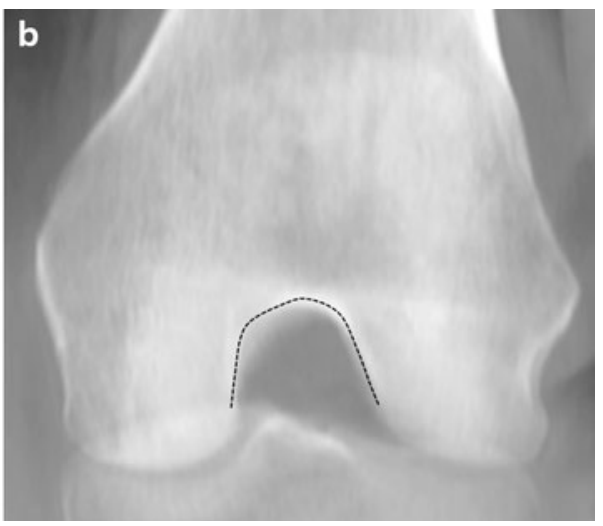

since the walls of the notch are relatively straight. b A-shaped notch; variation in the measurement location in this specimen would probably cause a large difference in notch width index, since the walls of the notch are sloped 
measurement of the NWI and cadaveric measurements was reported earlier by Anderson et al. [1]. We used the measurements of one observer for further statistical analysis, however, since a satisfactory correlation was seen between the observers, it was deemed appropriate. Lastly, due to that this was the first study to determine the correlation between the NWI and three-dimensional notch volume, we were unable to perform a reliable sample size calculation before conducting the study. This was mainly caused by the inability to predict the magnitude of the correlation between the NWI and notch volume, if any, which was the primary aim of the present study. However, with the results of our study, we have conduction a post hoc power calculation. With the correlation of the present study between $0.03(P 0.90)$ and $0.19(P 0.42)$, and a sample size of 20 specimens, the power is between 75 and $92 \%$, which was deemed appropriate.

Based on the main findings of the present study, we would recommend using caution when applying notch view radiographs in a clinical setting to predict risk of ACL rupture. Although all tested notch view radiographs proved reliable to measure NWI, there is no indication that NWI predicts overall notch size.

\section{Conclusion}

In conclusion, this study showed that there is no significant correlation between the NWI, as measured on three most frequently used notch view radiographs, and the overall volume of the notch as measured on CT scan. All three radiographic views, the Holmblad $45^{\circ}$, Holmblad $70^{\circ}$, and Rosenberg view, proved to be reliable, but showed only a moderate correlation with each other. Men had a significantly larger notch volume than women, but there was no difference in NWI.

Acknowledgments The authors would like to thank J. J. Irrgang, $\mathrm{PT}, \mathrm{PhD}, \mathrm{ATC}$, FAPTA, and S. Tashman, $\mathrm{PhD}$, for their helpful discussion. The authors did not receive any outside funding or grants directly related to the research described in this manuscript. Our institution receives a research grant from Smith and Nephew to support research related to ACL reconstruction. The research that forms the basis for this manuscript was done at our institution.

Open Access This article is distributed under the terms of the Creative Commons Attribution Noncommercial License which permits any noncommercial use, distribution, and reproduction in any medium, provided the original author(s) and source are credited.

\section{References}

1. Anderson AF, Anderson CN, Gorman TM, Cross MB, Spindler KP (2007) Radiographic measurements of the intercondylar notch: are they accurate? Arthroscopy 23:261-268

2. Chandrashekar N, Slauterbeck J, Hashemi J (2005) Sex-based differences in the anthropometric characteristics of the anterior cruciate ligament and its relation to intercondylar notch geometry: a cadaveric study. Am J Sports Med 33:1492-1498

3. Charlton WP, St John TA, Ciccotti MG, Harrison N, Schweitzer M (2002) Differences in femoral notch anatomy between men and women: a magnetic resonance imaging study. Am J Sports Med 30:329-333

4. Davis TJ, Shelbourne KD, Klootwyk TE (1999) Correlation of the intercondylar notch width of the femur to the width of the anterior and posterior cruciate ligaments. Knee Surg Sports Traumatol Arthrosc 7:209-214

5. Dienst M, Schneider G, Altmeyer K, Voelkering K, Georg T, Kramann B, Kohn D (2007) Correlation of intercondylar notch cross sections to the ACL size: a high resolution MR tomographic in vivo analysis. Arch Orthop Trauma Surg 127:253-260

6. Herzog RJ, Silliman JF, Hutton K, Rodkey WG, Steadman JR (1994) Measurements of the intercondylar notch by plain film radiography and magnetic resonance imaging. Am J Sports Med 22:204-210

7. LaPrade RF, Burnett QM 2nd (1994) Femoral intercondylar notch stenosis and correlation to anterior cruciate ligament injuries. A prospective study. Am J Sports Med 22:198-202

8. Lund-Hanssen H, Gannon J, Engebretsen L, Holen KJ, Anda S, Vatten L (1994) Intercondylar notch width and the risk for anterior cruciate ligament rupture. A case-control study in 46 female handball players. Acta Orthop Scand 65:529-532

9. Murshed KA, Cicekcibasi AE, Karabacakoglu A, Seker M, Ziylan T (2005) Distal femur morphometry: a gender and bilateral comparative study using magnetic resonance imaging. Surg Radiol Anat 27:108-112

10. Norwood LA Jr, Cross MJ (1977) The intercondylar shelf and the anterior cruciate ligament. Am J Sports Med 5:171-176

11. Shelbourne KD, Davis TJ, Klootwyk TE (1998) The relationship between intercondylar notch width of the femur and the incidence of anterior cruciate ligament tears. A prospective study. Am J Sports Med 26:402-408

12. Souryal TO, Freeman TR (1993) Intercondylar notch size and anterior cruciate ligament injuries in athletes. A prospective study. Am J Sports Med 21:535-539

13. Souryal TO, Moore HA, Evans JP (1988) Bilaterality in anterior cruciate ligament injuries: associated intercondylar notch stenosis. Am J Sports Med 16:449-454

14. Tillman MD, Smith KR, Bauer JA, Cauraugh JH, Falsetti AB, Pattishall JL (2002) Differences in three intercondylar notch geometry indices between males and females: a cadaver study. Knee 9:41-46 